\title{
Probiotics Supplements for the Management of Calcium Oxalate Kidney Stones-Are We Ready for it?
}

\section{Pragasam Viswanathan*}

Renal Research Lab, School of Biosciences and Technology, Centre for Biomedical Research, Vellore Institute of Technology, 632014, Vellore, Tamil Nadu, India

${ }^{*}$ Corresponding author: Pragasam Viswanathan, Renal Research Lab, School of Biosciences and Technology, Centre for Biomedical Research, Vellore Institute of Technology, 632014, Vellore, Tamil Nadu, India, Tel: +914162202583; E-mail: pragasam.v@vit.ac.in

Received Date: Apr 4, 2018; Accepted Date: Apr 18, 2018; Published Date: Apr 23, 2018

Copyright: (C) 2018 Viswanathan P. This is an open-access article distributed under the terms of the Creative Commons Attribution License, which permits unrestricted use, distribution, and reproduction in any medium, provided the original author and source are credited.

\section{Editorial}

The origin of the word probiotic comes from the Greek word "pro" meaning "promoting" and "biotic" meaning "life". The discovery of probiotics came about in the early $20^{\text {th }}$ century, when Elie Metchnikoff, known as the "Father of Probiotics" had observed that rural dwellers in Bulgaria lived to very old ages despite extreme poverty and harsh climate. In October 2013, the International Scientific Association for Probiotics and Prebiotics (ISAPP) define probiotics as "live microorganisms that, when administered in adequate amounts, confer a health benefit on the host".

Crystals of calcium oxalate are the most widely recognized kind of kidney stones. Kidney stones are dense masses that form in the kidney when there are increased levels of calcium, oxalate, cystine, or phosphate and very less liquid. Calcium oxalate stones are triggered by too much oxalate in the urine. Oxalate is considered an unusable end product of mammalian metabolism and urinary oxalate is derived from endogenous metabolic sources, primarily produced by the liver [1]. Almost $80-90 \%$ of kidney stone forming individuals form calcium oxalate stones and hyperoxaluria is a main risk factor in this kidney stone diseases.

One possible approach to prevent renal stone recurrence is to decrease the consumption of oxalate rich foods. However, albeit such dietary limitation has been accounted to decrease stone recurrence, its long term effectiveness is uncertain. Therefore, other methods aimed to reduce intestinal oxalate absorption have been sought. Both epithelial barriers of the intestine and kidney mediate oxalate balance. Several investigations acknowledged that intestinal commensal bacteria with oxalate degrading activity have the potential to contribute to oxalate homeostasis. Studies have demonstrated that rehashed use of antibiotics can result in the loss of naturally occurring oxalate-degrading bacteria. With this loss, dietary and endogenous oxalate turns out to be more bioavailable both to the mammalian host and intestinal microbiota.

The oxalate tolerance by the human beings and other mammals are often regularly encouraged by the presence of oxalate-degrading microorganisms in their intestinal microbiota. One of the first oxalatedegrading intestinal microbes to be characterized was Oxalobacter formigenes as it requires oxalate as a carbon and energy source. In addition $\sim 18$ other probiotics have been screened to degrade oxalate but do not require oxalate for its growth. These include species from the genera Lactobacillus, Bifidobacterium, Enterococcus, and Eubacterium among others [2]. O. formigenes contains a bunch of oxalate degrading enzymes such as oxalate oxidase, oxalate decarboxylase, oxalyl-CoA decarboxylase, and formyl-CoA transferase, which degrade oxalate into formate and carbon-di-oxide. The study conducted by Anbazhagan et al. developed a recombinant $L$. plantarum NC8 that constitutively expressed heterologous oxalate decarboxylase and degraded oxalate efficiently under in vitro conditions [3].

Studies conducted by both Sidhu et al. and Hatch et al. on experimentally induced hyperoxaluric animals with extraneous supplementation of $O$. formigenes daily for two weeks exhibited a $39-80 \%$ reduction in excreted urinary oxalate [4,5]. Likewise, humans supplemented with $O$. formigenes for four weeks exhibited a $22-92 \%$ reduction in urinary oxalate content. In addition to $O$. formigenes, a few lactobacilli and bifdo-bacteria represent potential contender for use in the anticipation and treatment of hyperoxaluria. One of the first studies on probiotic supplementation to human brings provided very promising results, where urinary and fecal oxalate levels were reduced in patients with idiopathic hyperoxaluria after treatment with a mixture of three Lactobacillus strains (L. acidophilus, L. plantarum and $L$. brevis), together with $B$. infantis and Streptococcus thermophilus [6]. The clinical trial conducted by Lieske et al. at Mayo Clinic, Rochester (US) used two probiotic preparations (Agri-King Synbiotic and Oxadrop) on urinary oxalate excretion in patients with mild hyperoxaluria. Probiotics supplements and placebo were given to patients for 4 weeks and found no change from baseline for any of the groups. The study finally concluded that probiotics did not influenced urinary oxalate levels in patients on a restricted oxalate diet. Rather, this investigation recommends that dietary oxalate limitation is helpful for kidney stone counteractive action $[7,8]$.

Google search indicated several such indigenous probiotics supplements are available in the market for people consumption, without proper validation and analysis. The major drawback concerned with all these products could be the use of freeze dried microbes or granules. $O$. formigenes is a strict anaerobic organism and all other probiotics are facultative and an aerobic organism, coadministrating these microbes as consortia requires numerous investigations. It is imperative to check its cell viability and intestinal colonization, because of low $\mathrm{pH}$ of the stomach and the presence of bile salts in the small intestine are the main reasons for the dramatic decline in viability of delivered cells [9-11]. Despite the fact that various advantages and health benefits are related with probiotics or probiotic food items, but also there are few risks that are related with probiotic treatment. Probiotics can interact with commensal microbes and can likewise directly affect the host. Understanding these collaborations is one of the key difficulties for future research.

Nutraceutical market indicated that probiotics are a big and rapidly growing business, with annual global sales of products expected to rise to $\$ 42$ billion by 2016 . Most probiotics are sold as dietary supplements; this means that the companies that make them don't have to test their products and show that they work or that they are safe, so more research is required to confirm that probiotics are safe and effective 
Citation: Viswanathan P (2018) Probiotics Supplements for the Management of Calcium Oxalate Kidney Stones-Are We Ready for it?. J Kidney

Page 2 of 2

and do not have any side effects on the individuals who are consuming those probiotics. In order improve the efficacy of probiotics; investigations are moving towards targeted delivery of probiotics to enhance the gastrointestinal stability and intestinal colonization. Dodoo et al. conducted a study on $L$. acidophilus after freeze drying and loaded into capsules for site specific delivery into the colon using the floral coating technology. The results confirmed that encapsulated organisms achieved viabilities of approximately $90 \%$ in the gastrointestinal tract and these organisms were adhered to the intestinal wall in the range of $64-76 \%$ for the products. The study successfully delivered the probiotics in the upper colon region, which become essential for success of probiotics as health supplements [12].

To conclude, the probiotics supplements are now broadly used as nutraceuticals and as health supplements. Accessible probiotics seems to be safe but should be avoided for the patients those are at high risk of septicemia and other clinical complications. Therefore, healthcare regulators and investigators should consider few points such as nutraceuticals should be produced with international regulations, advanced studies need to conduct for target delivery to avoid unnecessary complications, probiotics should not contain any virulence, side effects, resistance genes or antibiotics resistance genes and should offer characterized health benefits to the individuals.

\section{References}

1. Sridharan B, Ganesh RN, Viswanathan P (2016) Polyacrylic acid attenuates ethylene glycol induced hyperoxaluric damage and prevents crystal aggregation in vitro and in vivo. Chem Biol Interact 252: 36-46.

2. Liebman M, Al-Wahsh IA (2011) Probiotics and other key determinants of dietary oxalate absorption. Adv Nutr 2: 254-260.
3. Anbazhagan K, Sasikumar P, Gomathi S, Priya HP, Selvam GS (2013) In vitro degradation of oxalate by recombinant Lactobacillus plantarum expressing heterologous oxalate decarboxylase. J Appl Microbiol 115: 880-887.

4. Sidhu H, Allison MJ, Chow JM, Clark A, Peck AB (2001) Rapid reversal of hyperoxaluria in a rat model after probiotic administration of Oxalobacter formigenes. J Urol 166: 1487-1491.

5. Hatch M, Cornelius J, Allison M, Sidhu H, Peck A, et al. (2006) Oxalobacter spp. reduces urinary oxalate excretion by promoting enteric oxalate secretion. Kidney Int 69: 691-698.

6. Campieri C, Campieri M, Bertuzzi V, Swennen E, Matteuzzi D, et al. (2001) Reduction of oxaluria after an oral course of lactic acid bacteria at high concentration. Kidney Int 6: 1097-1105.

7. Lieske JC, Tremaine WJ, De Simone C, O'Connor HM, Li X, et al. (2010) Diet, but not oral probiotics, effectively reduces urinary oxalate excretion and calcium oxalate super saturation. Kidney Int 78: 1178-1185.

8. Lieske JC (2017) Probiotics for prevention of urinary stones. Ann Transl Med 5: 29 .

9. Tamime AY, Saarela M, Korslund SA, Mistry VV, Shah N (2005) Production and maintenance of viability probiotics micro-organism in dairy products: pp 39-72.

10. Kosin B, Rakshit SK (2006) Microbial and processing criteria for production of probiotics: A review. Food Technol Biotechnol 44: 371-379.

11. Korbekandi H, Mortazavian AM, Iravani S (2011) Technology and stability of probiotic in fermented milks containing probiotics and prebiotics. Nova Science Publishers, USA.

12. Dodoo CC, Wang J, Basit AW, Stapleton P, Gaisford S (2017) Targeted delivery of probiotics to enhance gastrointestinal stability and intestinal colonisation. Int J Pharm 530: 224-229. 